УДК ОО $4+658$

$10.17213 / 2075-2067-2020-6-224-233$

\title{
СОКРАЩЕНИЕ БЮДЖЕТНЫХ АССИГНОВАНИЙ СУБЪЕКТОВ РЫНКА ПУТЕМ УНИФИКАЦИИ ДЕЛОВЫХ ПРОЦЕССОВ ОДНОГО НАЗНАЧЕНИЯ ${ }^{1}$
}

\author{
(C) 2020 г. Г. Н. Хубаев
}

\section{Ростовский государственный экономический университет (РИНХ), 2. Ростов-на-Дону, Россия}

Целью исследования является разработка методики и инструментальных средств для оценки экономической целесообразности унификаџии деловых процессов одного назначения.

Методологическую базу исследования представляют экономическая теория, идеи и рекомендации по совершенствованию рыночной экономики, содержащиеся в работах Мориса Алле (M. Allais) и Фредерика Хайека (F. Hayek), экономико-математические методы и модели.

Результаты исследования. Создано методическое и инструментальное обеспечение расчетов экономического эффекта от унификации деловых прочессов одного назначения. Обосновано использование визуализаџии для "наглядного представления деловых прочессов одного назначения, "автоматизированного преобразования визуальных моделей в имитационные модели, "сокращения финансовых и трудовых затрат на оценку ресурсоемкости и себестоимости выполнения деловых прочессов. Разработана оригинальная компьютерная программа для сравнительной оценки состава операчий деловых прочессов одного назначения, предложен метод количественной оченки взаимосвязи по операчиям между деловыми процессами, позволяющий выявить и исключить дублирование прочессов и операций.

Перспектива исследования состоит в том, что созданное в проиессе исследований методическое и инструментальное обеспечение расчетов экономического эффекта от унификации деловых процессов одного назначения позволяет субъектам рынка целенаправленно сокращать бюджетные ассигнования, содействовать росту сочиильно устойчивой экономики.

Ключевые слова: деловые процессы; унификация; реинжиниринг; имитационное моделирование; автоматизированный синтез; визуализачия; экономическая эффективность.

\section{REDUCTION OF BUDGET ALLOCATIONS OF MARKET ENTITIES BY UNIFYING BUSINESS PROCESSES FOR A SINGLE PURPOSE}

\author{
(C) 2020 G. N. Khubaev
}

\section{Rostov State University of Economics (RSUE), Rostov-on-Don, Russia}

The purpose of the research is to develop methods and tools for assessing the economic feasibility of unifying business processes for one purpose.

1 Статья подготовлена по результатам исследований, выполненных при поддержке Российского фонда фундаментальных исследований (РФФИ) — проект 15-01-06324/15 «Моделирование производственных и управленческих процессов для экспресс-оценки и оптимизации ресурсоемкости товаров и услуг: формирование универсального методического и инструментального обеспечения». 
The methodological basis of the research is economic theory, ideas and recommendations for improving the market economy contained in the works of Maurice Allais and Frederick Hayek, economic and mathematical methods and models.

Research result. Methodological and instrumental support for calculating the economic effect of the unification of business processes for one purpose has been created. The use of visualization is justified for ${ }^{*}$ visual representation of business processes of a single purpose, *automated conversion of visual models into simulation models, "reduction of financial and labor costs for assessing the resource intensity and cost of business processes. Developed an original computer program for the comparative evaluation of the operations business processes one destination and proposed method of quantifying the linkage in the operations between the business processes to identify and eliminate duplication of processes and operations.

The research perspective is that the methodological and instrumental support for calculating the economic effect of the unification of business processes for one purpose created in the course of research allows market entities to purposefully reduce budget allocations and promote the growth of a socially sustainable economy.

Key words: business processes; unification; reengineering; simulation; automated synthesis; visualization; economic efficiency.

Введение. Напомним сначала, что «субъекты рынка: 1) физические лица; 2) юридические лица; 3) государство» [1]. При этом государство и местные бюджеты финансируют деятельность не только государственного аппарата и органов управления, но и значительной части учреждений науки, культуры, образования и здравоохранения. Однако в процессе сравнительного экономико-математического анализа и моделирования деловых процессов (ДП) в перечисленных предметных областях обнаружен значительный разброс в структуре и количестве операций, в затратах времени, трудовых и финансовых ресурсов на выполнение ДП одного назначения (см., например, [2-8]); при этом одновременно с увеличением численности исполнителей любого делового процесса (технологического или управленческого) возрастают, как правило, и затраты материальных, энергетических и финансовых ресурсов.

Не менее существенная разница в ресурсоемкости ДП одного назначения и у многих юридических лиц - в крупных торговых сетях, холдингах, у филиалов банков, на предприятиях схожей производственной направленности нефтегазового сектора и у множества других объектов в различных предметных областях, включая медицину, образование и т.д. Действительно, как показали наши исследования, реализация проекта по реинжинирингу и унификации делового процесса одного назначения только на одном предприятии и только с частичной унификацией обеспечила существенную экономию бюджетных средств юридического лица, дала, судя по экономическим оценкам и по величине полученной исполнителем премии, весьма ощутимый прямой экономический эффект и позволила отказаться от дополнительного набора нескольких высококвалифицированных специалистов (см., например, [9]).

Да и физические лица - покупатели товаров и услуг вряд ли откажутся от сокращения расходной части своего бюджета, поскольку заинтересованы в минимизации расхода собственных бюджетных средств для обеспечения своей жизнедеятельности.

Получается, что все субъекты рынка заинтересованы в сокращении своих бюджетных ассигнований на реализацию процессов своего функционирования, поэтому, естественно, возникает ряд вопросов: Как получить количественное обоснование целесообразности и экономической эффективности унификации деловых (технологических и/или управленческих) процессов одного назначения? Какими методами и инструментальными средствами это можно осуществить? Какова величина экономии финансовых ре- 
сурсов субъекта рынка при унификации ДП одного назначения? Причем, в отечественной и зарубежной литературе отсутствуют ответы на эти вопросы.

В статье на базе ранее выполненных исследований $[10-20,22]$ предложены оригинальная методика и инструментальные средства для вылвления, оценки и обоснования возможности и экономической эффективности унификации деловых процессов одного назначения.

\section{1. Почему субъектам рынка выгодна} унификация ДП одного назначения. Для оценки на качественном уровне экономической полезности осуществления унификации ДП одного назначения проведем содержательный анализ наличия выгоды для субъектов рынка от реализации подобных проектов.

"Bыгоды государства. Государство, оптимизировав бюджетные ассигнования за счет унификации технологических и управленческих процессов одного назначения, сможет направить «освободившиеся» финансовые ресурсы на другие требующие внимания нужды, например, в социальную сферу, обеспечивая субсидирование различных социальных выплат.

"Выгоды юридических лиц. Не меньшими возможностями для сокращения своих бюджетных ассигнований и для минимизации издержек производственной деятельности располагают и многие юридические лица - торговые сетевые компании («Перекресток», «Пятерочка», «Лента» и др.), крупные холдинги, банки с множеством филиалов, управляющие компании в ЖКХ и др.

Так, получив за счет унификации технологических и/или управленческих процессов $\partial о-$ полнительно определенный объем финансовых ресурсов, юридчческое лицо укрепит свои конкурентные рыночные позиции и сможет (по аналогии с действиями многих японских и китайских компаний) в процессе конкурентной борьбы снизить цены производимых товаров и предоставляемых услуг, обеспечивая более высокий уровень конкурентоспособности результатов своей производственной деятельности - товаров и услуг.

Действительно, как показывают исследования на реальных объектах, прямой экономический эффект от реинжиниринга и уни- фикации ДП одного назначения может быть весьма значительным [9].

"Выгоды физических лиц. Что касается физических лиц, то можно предположить, что реальная, но не всегда осознанная заинтересованность населения страны, гражданпокупателей товаров и услуг в унификации государством и юридическими лицами всех технологических и управленческих процессов одного назначения достаточно велика, поскольку, во-первых, государство освободившиеся ресурсы сможет направить на материальную поддержку беднейших групп населения или на разовые доплаты бюджетникам, а во-вторых, что весьма вероятно, юридические лица, получив финансовую «подушку безопасности» в результате унификации своих ДП одного назначения, будут гораздо чаще использовать ценовую конкуренцию, то есть снижать цену товара или услуги, одновременно поддерживая (осознанно или нет) рост сочиально устойчивой экономики.

Вывод. Содержательный анализ ситуации на рынке товаров и услуг неожиданно показал, что унификация ДП одного назначения позволяет всем субъектам рынка сократить бюджетные ассигнования и оптимизировать свои расходы. Причем, по мере того, как государство и юридические лища будут реализовывать проекты унификации и реинжиниринга ДП, эти проекты и действия окажут позитивное влияние и на жизнедеятельность физических лиц, на население страны, на граждан - покупателей товаров и услуг.

2. Сравнительная количественная оценка экономического эффекта от унификации деловых процессов одного назначения.

"Используемые обозначения. Количество анализируемых деловых процессов обозначим буквой $N,(j \in N)$, а количество операций в составе $\boldsymbol{j}$-го ДП $-\boldsymbol{n}^{(j)}$. Пусть $\boldsymbol{N}=100$. Отдельная $\boldsymbol{i}$-я операция в составе $\boldsymbol{j}$-го процесса $-\boldsymbol{o}^{(j)}{ }_{i}$. Cебестоимость (трудоемкость, ресурсоемкость) $\boldsymbol{j}$-го процесса $-\boldsymbol{S}^{(j)}$, а себестоимость $\boldsymbol{i}$-й операции $\boldsymbol{j}$-го процесса $-\boldsymbol{S}^{(j)}{ }_{i}$. Изменение (прирост) себестоимости $\boldsymbol{j}$-го ДП $-\Delta \boldsymbol{S}^{(j)}$. Первый, второй, ..., девятый децили: $\boldsymbol{D Z 1}, \boldsymbol{D Z 2}, \ldots$, DZ9. Количество выполнений $\boldsymbol{j}$-го делового процесса в течение месяца - $\boldsymbol{k}_{\text {мес }}$, а года $\boldsymbol{k}_{\text {zød }}^{\boldsymbol{j}}$. Оценка себестоимости выполнения $\boldsymbol{j}$-го делового процесса 


$$
S^{(j)}=\sum S_{i}^{(j)} \times n^{(j)} ;(j \in N),\left(i \in n^{(j)}\right) .
$$

Количественная оценка годового эффекта от унификации $\boldsymbol{j}$-го делового процесса

$$
\Delta \boldsymbol{S}^{(j)}{ }_{2 o d}=\sum \Delta \boldsymbol{S}^{(j)} \times \boldsymbol{k}_{\text {Mec }}^{j} .
$$

\section{*Предлагаемая последовательность шагов.}

Шаг 2.1. Выбор делового процесса для исследования. Предполагается, что этот процесс активно используется государством или юридическим лицом во многих учреждениях конкретного направления (в конкретной отрасли, конкретной предметной области).

Шаг 2.2. Формирование базы исходной информации, содержащей сведения о количестве выполнений этого ДП по дням месяца и/или по месяцам за предыдущий год в каждом из учреждений в рассматриваемой предметной области.

Шаг 2.3. Оценка статистических характеристик (вычисляются математическое ожидание, дисперсия, стандартное отклонение, коэффициент вариации, асимметрия, эксцесс), таблиц и гистограмм распределения значений количества выполнений ДП одного назначения в каждом из $\boldsymbol{j},(\boldsymbol{j} \in \boldsymbol{N})$ рассматриваемых учреждений в течение месяца $-\boldsymbol{k}_{\text {мес }}^{j}$ и года $-\boldsymbol{k}^{j}$.od.

Шаг 2.4. Осуществляется сравнительная количественная оценка состава операций по всем изучаемым ДП. Одновременно выполняется оценка взаимосвязи по операциям между ДП одного назначения с использованием программной системы [21].

Шаг 2.5. Определение затрат финансовых ресурсов на однократное выполнение всех операций каждого $\boldsymbol{j}$-го ДП и себестоимости выполнения каждого из $N(j \in N)$ ДП в целом. Для этого необходимо оценить пооперационные затраты времени, трудовых ресурсов (количество исполнителей ДП), величину заработной платы исполнителей, затраты материалов, их стоимость и стоимость используемых технических средств (для оценки величины амортизационных отчислений).

Шаг 2.6. Визуализация каждого из анализируемых деловых прочессов. Визуализация позволяет осуществить *наглядное представление деловых процессов одного назначе- ния, *описать концепции предметной области и *реализовать последующий автоматизированный синтез имитационных моделей, что обеспечивает многократное сокращение финансовых и трудовых затрат на выполнение прочедур, связанных с оценкой себестоимости выполнения и ресурсоемкости деловых процессов (см., например, [10-15]).

Шаг 2.7. Оценка статистических характеристик (математического ожидания, дисперсии, стандартного отклонения, коэффициента вариации, асимметрии, эксцесса), таблиц и гистограмм распределения затрат финансовых ресурсов [16-20, 23] на однократное выполнение каждого из сравниваемых деловых процессов одного назначения (по себестоимости однократного выполнения каждой операции делового процесса):

$$
S^{(j)}=\sum S_{i}^{(j)} \times n^{(j)}(j \in N),\left(i \in n^{(j)}\right) .
$$

Шаг 2.8. Сортировка всех ДП по возрастанию затрат финансовых ресурсов на выполнение ДП (по возрастанию средней величины себестоимости ДП или по значению, которое соответствует затратам на выполнение ДП с вероятностью 0,$8 ; 0,9 ; \ldots)$. Упорядоченную по себестоимости однократного выполнения совокупность ДП одного назначения делят на десять равных частей $\boldsymbol{n} / \mathbf{1 0}, \mathbf{2 n} / \mathbf{1 0}, \ldots$, 9n/10 - децилей: $D Z 1, D Z 2, \ldots, D Z 9$.

Шаг 2.9. Определение средней себестоимости однократного выполнения ДП в первом дециле $\boldsymbol{D Z 1}-\boldsymbol{S}^{\boldsymbol{D Z 1}}$.

Шаг 2.10. Вычисление разницы между затратами финансовых ресурсов на однократное выполнение ДП одного назначения в первом дециле $\boldsymbol{D Z} \mathbf{Z 1}$ и в децилях $\boldsymbol{D Z Z 2 , . . . ,}$ DZ9.

$$
\begin{gathered}
\Delta S^{(11)}=\left(S^{(11)}-S^{D Z 1}{ }_{\mathrm{cp}}\right), \\
\Delta S^{(12)}=\left(S^{(12)}-S^{D Z 1}{ }_{\mathrm{cp}}\right), \ldots, \\
\Delta S^{(91)}=\left(S^{(91)}-S^{D Z 1}{ }_{\mathrm{cp}}\right), \ldots, \\
\Delta S^{(100)}=\left(S^{(100)}-S^{D Z 1}{ }_{\mathrm{cp}}\right) .
\end{gathered}
$$

Шаг 2.11. Вычисление значений возможного годового сокращения бюджетных ассигнований (сокращения расходной части бюджета в течение года - оценка «снизу», по минимуму) в каждом из $\boldsymbol{j},(\boldsymbol{j} \in \boldsymbol{N})$ учреждений анализируемой предметной области (отрасли народного хозяйства и т.д.) путем 
имитационного моделирования с использованием системы автоматизированного синтеза имитационных моделей:

$$
\Delta \boldsymbol{S}_{\text {год }}^{(j)}=\sum \Delta \boldsymbol{S}^{(j)} \times \boldsymbol{k}_{\text {мec }}^{j}
$$

Шаг 2.12. Экспресс-оценка «снизу» (по минимуму) суммарной величины экономии финансовых ресурсов субъектами рынка (в результате оптимизации финансовых расходов) при унификации делового процесса одного назначения (из достаточно большой группы подобных ДП в странах мира и административно-территориальных образованиях). С этой целью выполняется имитационное моделирование произведения величины прироста себестоимости выполнения $\boldsymbol{j}$-го ДП на фактическое распределение количества выполнений в течение года $\boldsymbol{k}_{\text {гол }}$ анализируемого делового проекта в каждом из $\boldsymbol{j},(\boldsymbol{j} \in \boldsymbol{N})$ учреждений:

$$
\Delta \boldsymbol{S}_{\text {общ }}=\sum \Delta \boldsymbol{S}_{\text {год }}^{(j)} \times \boldsymbol{k}_{\text {год }}^{j},(j \in N) .
$$

[Замечание. Для получения более точной оценки экономического эффекта от унификации ДП одного назначения необходимо: * выявить и ранжировать факторы - классификациионые признаки, оказывающие основное влияние на рост себестоимости анализируемых ДП (см., например, [16, 22]); " выполнить сортировку всех $\boldsymbol{N}$ анализируемых процессов по возрастанию себестоимости выполнения (исходный вариант оценки себестоимости при фактическом количестве выполнений процессов и операций); "выполнить классификацию исходного множества ДП по выделенным признакам для получения квази-однородных, схожих по условиям реализации подмножеств; *отсортировать каждый класс по возрастанию значений себестоимости ДП; * рассчитать разность между средней себестоимостью по классу и средней себестоимостью в первом дециле этого класса; "получить величину экономического эффекта от унификации ДП в этом классе, умножив результат (вычисленное значение разности) на количество ДП в классе; * сложить значения экономического эффекта, рассчитанные по каждому классу для оценки экономического эффекта от унификации всех первоначально выбранных исследователем ДП одного назначения в конкретной предметной области.]
3. Оценка экономической целесообразности перманентного реинжиниринга и унификации деловых процессов одного назначения: предлагаемая последовательность шагов. После выявления резервов снижения себестоимости выполнения $u$, возможно, полной ресурсоемкости деловых процессов одного назначения в условия $\boldsymbol{x}$ применения существующей технологии целесообразно * выполнить анализ возможности и экономической обоснованности перехода к использованию новых, менее ресурсоемких процессов и технологий, "оценить экономическую целесообразность реинжиниринга u унификации выбранной группы деловых процессов одного назначения (на определенное время, в целом по стране или в нескольких административно-территориальньх образованиях).

С целью минимизации себестоимости (ресурсоемкости) деловых процессов одного назначения реализуются следующие шаги.

Шаг 3.1. Выполняется поиск (в paзных странах и административно-территориальных образованиях страны) процессов с одинаковым иелевым назначением (с одинаковой целевой функцией) и со схожими условиями осуществления или со схожим функииональным назначением отдельных операичuй.

Шаг 3.2. Формируется база данных, включающая подмножество альтернативных деловых процессов, отличаюшихся составом, содержанием или временем выполнения и трудоемкостью (ресурсоемкостью) отдельных операций.

Шаг 3.3. Выполняется количественная оценка степени взаимосвязи по операциям между всеми сущзествующими и проектируемыли процессами, включенными в базу данных [21], и сравнительный анализ трудоемкости (себестоимости, ресурсоемкости) одинаковых операций при выполнении процессов в разных странах и в разных административно-территориальных образованиях страны.

[Замечание. Если в базу данных включить все известные процессы с одинаковым целевым назначением, но с разнылм условиями осуществления, то в последующем, сформировав репрезентативную выборку и построив экономико-математические моде- 
ли, можно количественно оченить степень влияния условий осуществления делового процесса на величину его ресурсоемкости.]

Шаг 3.4. Выполняют оценку себестоимости выполнения или ресурсоемкости каждой операции по всем процессам, включенным в сформированную базу данных (используются процессно-статистический метод учета затрат ресурсов [10] и метод пошагового уточнения значений затрат с оценкой характеристик распределения $[11,12])$.

Шаг 3.5. Выполняется оченка резервов снижения затрат ресурсов на реализацию отдельных операций, различных подмножеств операций и процесса в целом. Для объективной количественной оценки резервов снижения затрат трудовых и финансовых ресурсов в результате реализачии проекта реинжиниринга определяют степень влияния ресурсоемкости (трудоемкости, себестоимости) каждой операции на статистические характеристики распределения значений ресурсоемкости делового процесса.

Шаг 3.6. Выполняют: "сравнительную количественную оценку разницы в затратах ресурсов на выполнение одинаковых операций; "обоснованный (оптимальный) выбор подходящего процесса из множества сопоставимых (по критерию минимума затрат ресурсов, например, труда, финансов и времени на получение конкретных результатов или по критерию минимума совокупной стоимости владения процессом [17]); "разработку рекомендаций по оптимизачии численности персонала, реализующего деловой проиесс; "оценку резервов снижения затрат ресурсов при выборе (по критерию минимума ресурсоемкости) делового процесса по сравнению с анализируемым процессом (существуюшим, включенным в созданную базу данных или проектируемылм); "сравнительный анализ эффективности возможных вариантов реинжиниринга и унификации выбранного делового процесса, включая, например, реализацию услуг населению.

Выводы. В процессе исследований впервые:

1. Разработана методика оценки экономической целесообразности унификащии деловых процессов одного назначения, ориентированная на формирование базы данных, включа- ющей сведения об альтернативных деловых процессах, отличающихся составом, содержанием или временем выполнения и ресурсоемкостью отдельных операций. При наличии такой базы данных можно осуществлять обоснованный (оптимальный) выбор подходящего делового процесса из множества сопоставимых (по критерию минимума затрат финансовых ресурсов или минимума совокупной стоимости владения деловым процессом) и оценивать резервы снижения затрат ресурсов при выборе конкретного делового процесса.

2. Обосновано использование визуализации для *наглядного представления деловых процессов одного назначения, *описания концепций предметной области, *автоматизированного преобразования визуальных моделей в имитационные модели, "сокращения финансовых и трудовых затрат на оценку ресурсоемкости и себестоимости выполнения деловых процессов. Создана оригинальная компьютерная программа для сравнительной оценки состава операций деловых процессов одного назначения и предложен метод количественной оценки взаимосвязи по операциям между деловыми процессами, позволяющий выявить и исключить дублирование процессов и операций.

3. В процессе исследований сформировано методическое и инструментальное обеспечение расчетов экономического эффекта от унификации деловых процессов одного назначения, позволяющее субъектам рынка целенаправленно сокращать бюджетные ассигнования, содействуя росту социально устойчивой экономики.

\section{Литература}

1. Большая экономическая энциклопедия. - М.: Эксмо, 2007. - 816 с.

2. Система поддержки принятия решений для контроля правильности исчисления налога на прибыль (СППР «Налоговый учет. 1.0») / А.Б. Паскачев, Ю.Д. Джамурзаев, Г.Н. Хубаев, О. В. Родина / Под ред. А. Б. Паскачева и Г.Н. Хубаева. - М.: Изд-во экономико-правовой литературы, 2004. - 120 с.

3. Моделирование деловых процессов в налоговых инспекциях / А.Б. Паскачев, Ю.Д. Джамурзаев, Г.Н. Хубаев, С.Н. Широбокова / Под общ. ред. Т.В. Шевцовой, 
Д. А. Чушкина. - М.: Изд-во экономико-правовой литературы, 2006. - 304 с.

4. Родина О.В. Налоговый учет: экономико-математические модели, методы и программные средства для оценки и минимизации затрат ресурсов на ведение и мониторинг: монография. - М.: ФГБОУ ВПО «РЭУ им. Г.В. Плеханова», 2011. - 144 с.

5. Хубаев Г.Н., Родина О.В. Математические методы при оценке налогового потенциала предприятий по значениям косвенных факторов // Обозрение прикладной и промышленной математики. - М.: Редакция журнала «ОП и ПМ», 2005. — Т. 12. Вып. 2. - С. 555-557.

6. Родина О.В. Методика рейтинговой оценки налоговых органов по результатам внутреннего аудита // Налоги и финансы. 2018. - №2. - C. 45-54.

7. Хубаев Г.Н., Бастрыкина М.А., Калугян К.Х., Щербаков С.М. Экспресс-оценка и минимизация ресурсоемкости деловых процессов в вузе // Российский экономический интернет-журнал. - 2017. - №4. C. 69.

8. Хубаев Г.Н., Широбокова С.Н., Бабеев М.C. Сравнительный анализ ресурсоемкости процессов бухгалтерского учета в учреждениях высшего образования // Российский экономический интернет-журнал. 2018. - №1. - C. 37.

9. Хубаев Г.Н., Ким М.С. Реинжиниринг и унификация процессов, связанных с авариями и катастрофами для уменьшения социальных и экономических потерь // Sciences of Europe. - 2018. - Vol. 3. Economic Sciences. - №26. - P. 16-21.

10. Хубаев Г.Н. Калькуляция себестоимости продукции и услуг: процессно-статистический учет затрат // Управленческий учет. - 2009. - №2. - С. 35-46.

11. Хубаев Г.Н. Имитационное моделирование для получения групповой экспертной оценки значений различных показателей// Автоматизация и современные технологии. - 2011. - №11. - С. 19-23.

12. Хубаев Г.Н. Оценка резервов снижения ресурсоемкости товаров и услуг: методы и инструментальные средства // Прикладная информатика. - 2012. - №2. - С.84-90.

13. Хубаев Г.Н., Калугян К.Х., Родина О.В., Щербаков С.М., Широбокова С.Н. У Унвер- сальное методическое и инструментальное обеспечение экспресс-оценки и оптимизации ресурсоемкости товаров и услуг// Бюллетень науки и практики. Электронный журнал. 2016. 一 №12. - С. 286-299.

14. Хубаев Г.Н., Широбокова С.Н. Инструментарий преобразования IDEF3-моделей бизнес-процессов в UML-диаграммы // Глобальный научный потенциал. - 2015. №2. - С. 87-96.

15. Хубаев Г.Н., Широбокова С.Н. Визуальное и имитационное моделирование для экспресс-оценки ресурсоемкости технологических и управленческих процессов // Глобальный научный потенциал. - 2014. №6. - C. 60-66.

16. Хубаев Г.Н. Универсальный метод оптимизации состава характеристик объектов [Электронный ресурс] // Бюллетень науки и практики. - 2019. - Т. 5. - №5. С. 265-275. - Режим доступа: http://doi. org/10.33619/2414-2948/42/35.

17. Хубаев Г., Родина О. Модели, методы и программный инструментарий оценки совокупной стоимости владения объектами длительного пользования (на примере программных систем) / Монография. Saarbrucken: LAP LAMBERT Academic Publishing, 2012. - $370 \mathrm{c}$.

18. Система автоматизированного синтеза имитационных моделей на основе языка UML «CИM-UML» / Авторы-правообладатели: Хубаев Г.Н., Щербаков С.М., Рванцов Ю.А. // GeBIT 2015 (Ганновер, 2015). Каталог разработок российских компаний. Ministry of Education and Science of the Russian Federation; МСП ИТТ. - 2015.

19. Хубаев Г.Н., Щербаков С.М. Система автоматизированного синтеза имитационных моделей на основе языка UML 2.0 (CИM-UML 2.0) // Свидетельство о государственной регистрации программы для ЭВМ №2016661676. - М.: Роспатент, 2016.

20. Автоматизированный конвертер моделей IDEF0 в диаграммы деятельности языка UML «ToADConverter» («ToADConverter»)/ Авторы-правообладатели: Хубаев Г.Н., Широбокова С.Н., Ткаченко Ю.В., Титаренко Е. В. // GeBIT 2015 (Ганновер, 2015). Каталог разработок российских компаний. Ministry of Education and Science of the Russian Federation; МСП ИТТ. — 2015. 
21. Хубаев Г.Н., Щербаков С.М., Аручиди Н. А., Лубянский В.К. Сравнительная оценка состава операций деловых процессов (СОСОП) // Свидетельство о государственной регистрации программы для ЭВМ №2017614714. - М.: Роспатент, 2017.

22. Khubaev G. N. Method of isolating a desired subset of objects from a set of greater power [Electronic resource] // Scientific research of the SCO countries: synergy and integration (Beijing, China 31 July 2019). — Beijing, 2019. P. 50-57. — URL: https://doi.org/10.34660/ INF.2019.13.33962.

23. Khubaev G. N., Scherbakov S.M., Shirobokova S.N. Conversion of IDEF3 models into UML-diagrams for the simulation in the SIM system-UML // European Science Review. 2015. — №12. — P. 20-25.

\section{References}

1. Bol'shaja jekonomicheskaja jenciklopedija [The great economic encyclopedia]. - Moscow: Jeksmo, 2007. - 816 p.

2. Sistema podderzhki prinjatija reshenij dlja kontrolja pravil'nosti ischislenija naloga na pribyl' [The decision support System for controlling the correctness of calculating profit tax] (SPPR «Nalogovyj uchet. 1.0») / A.B. Paskachev, Ju. D. Dzhamurzaev, G. N. Hubaev, O. V. Rodina / In A. B. Paskachev and G. N. Hubaev (eds.). Moscow: Izd-vo jekonomiko-pravovoj literatury, 2004. - $120 \mathrm{p}$.

3. Modelirovanie delovyh processov $\mathrm{V}$ nalogovyh inspekcijah [Modeling of business processes in tax inspections] / A. B. Paskachev, Ju. D. Dzhamurzaev, G. N. Hubaev, S. N. Shirobokova / In T.V. Shevcova, D.A. Chushkin (eds.). - Moscow: Izd-vo jekonomiko-pravovoj literatury, 2006. - $304 \mathrm{p}$.

4. Rodina O.V. Nalogovyj uchet: jekonomikomatematicheskie modeli, metody i programmnye sredstva dlja ocenki i minimizacii zatrat resursov na vedenie i monitoring: monografija [Tax accounting: economic-mathematical models, methods and software for estimating and minimizing the cost of resources on maintenance and monitoring: monograph]. - Moscow: FGBOU VPO «RJeU im. G. V. Plehanova», 2011. — 144 p.

5. Hubaev G.N., Rodina O.V. Matematicheskie metody pri ocenke nalogovogo potenciala predprijatij po znachenijam kosvennyh faktorov [Mathematical methods in the assessment of the tax potential of the enterprises by the values of indirect factors] // Obozrenie prikladnoj i promyshlennoj matematiki [Review of applied and industrial mathematics]. - Moscow: Redakcija zhurnala «OP i PM», 2005. Vol. 12. - Issue 2. - Pp. 555-557.

6. Rodina O.V. Metodika rejtingovoj ocenki nalogovyh organov po rezul'tatam vnutrennego audita [Method of rating assessment of tax authorities based on the results of internal audit] // Nalogi i finansy [Taxes and Finance]. 2018. - №2. - Pp. 45-54.

7. Hubaev G.N., Bastrykina M.A., Kalugjan K.H., Shherbakov S.M. Jekspress-ocenka i minimizacija resursoemkosti delovyh processov $\mathrm{v}$ vuze [Rapid assessment and minimization of resource intensity in business processes in higher education] // Rossijskij jekonomicheskij internet-zhurnal [Russian economic Internet magazine]. — 2017. — №4. - Pp. 69.

8. Hubaev G.N., Shirobokova S.N., Babeev M.S. Sravnitel'nyj analiz resursoemkosti processov buhgalterskogo ucheta $\mathrm{v}$ uchrezhdenijah vysshego obrazovanija [Comparative analysis of the intensity of the processes of accounting in institutions of higher education]// Rossijskij jekonomicheskij internet-zhurnal [Russian economic online magazine]. - 2018. — №1. - Pp. 37.

9. Hubaev G.N., Kim M.S. Reinzhiniring i unifikacija processov, svjazannyh s avarijami i katastrofami dlja umen'shenija social'nyh i jekonomicheskih poter' [Reengineering and unification of processes related to accidents and catastrophes to reduce social and economic losses]// Sciences of Europe. - 2018. - Vol. 3. Economic Sciences. - №26. - P. 16-21.

10. Hubaev G.N. Kal'kuljacija sebestoimosti produkcii i uslug: processno-statisticheskij uchet zatrat [Costing of products and services: process-statistical cost accounting] // Upravlencheskij uchet [Management accounting]. 2009. - №2. - Pp. 35-46.

11. Hubaev G. N. Imitacionnoe modelirovanie dlja poluchenija gruppovoj jekspertnoj ocenki znachenij razlichnyh pokazatelej [Simulation to obtain the group expert estimation of values of various indicators] // Avtomatizacija i sovremennye tehnologii [Automation and modern technology]. — 2011. — №11. — Pp. 19-23.

12. Hubaev G. N. Ocenka rezervov snizhenija resursoemkosti tovarov i uslug: metody i 
instrumental'nye sredstva [Estimation of reserves for reducing the resource intensity of goods and services: methods and tools] // Prikladnaja informatika [Applied informatics]. 2012. 一 №2. - Pp.84-90.

13. Hubaev G.N., Kalugjan K.H., Rodina O.V., ShherbakovS.M., ShirobokovaS.N. Universal'noe metodicheskoe i instrumental'noe obespechenie jekspress-ocenki i optimizacii resursoemkosti tovarov i uslug [Universal methodological and instrumental support for Express assessment and optimization of resource intensity of goods and services]// Bjulleten' nauki i praktiki. Jelektronnyj zhurnal [Bulletin of science and practice. Electronic journal]. 2016. — №12. — Pp. 286-299.

14. Hubaev G.N., Shirobokova S.N. Instrumentarij preobrazovanija IDEF3-modelej biznesprocessov $\mathrm{v}$ UML-diagrammy [Tools convert IDEF3 models the business processes in UML diagrams] // Global'nyj nauchnyj potencial [Global scientific potential]. — 2015. — №2. - Pp. 87-96.

15. Hubaev G. N., Shirobokova S.N. Vizual'noe i imitacionnoe modelirovanie dlja jekspress-ocenki resursoemkosti tehnologicheskih i upravlencheskih processov [Visual modeling and simulation for rapid assessment of resource production and management processes]// Global'nyj nauchnyj potencial [Global scientific potential]. - 2014. - №6. - Pp. 60-66.

16. Hubaev G.N. Universal'nyj metod optimizacii sostava harakteristik obektov [Universal method for optimizing the composition of object characteristics] [Jelektronnyj resurs] // Bjulleten' nauki i praktiki [Bulletin of science and practice]. 2019. - Vol. 5. - №5. - Pp. 265-275. - URL: http://doi.org/10.33619/2414-2948/42/35.

17. Hubaev G., Rodina O. Modeli, metody i programmnyj instrumentarij ocenki sovokupnoj stoimosti vladenija obektami dlitel'nogo pol'zovanija (na primere programmnyh sistem) [Models, methods and software tools for assessing the total cost of ownership of long-term objects (on the example of software systems)] / Monografija. - Saarbrucken: LAP LAMBERT Academic Publishing, 2012. - 370 p.

18. Sistema avtomatizirovannogo sinteza imitacionnyh modelej na osnove jazyka UML
«SIM-UML» [System of automated synthesis of simulation models based on the UML language «SIM-UML»] / Authors-copyright holders: Hubaev G. N., Shherbakov S.M., Rvancov Ju. A. // GeBIT 2015 (Gannover, 2015). Katalog razrabotok rossijskih kompanij [Catalog of developments of Russian companies]. - Ministry of Education and Science of the Russian Federation; MSP ITT. - 2015.

19. Hubaev G.N., Shherbakov S.M. Sistema avtomatizirovannogo sinteza imitacionnyh modelej na osnove jazyka UML 2.0 [Computer-aided synthesis of simulation models based on UML 2.0] (SIM-UML 2.0) // Svidetel'stvo o gosudarstvennoj registracii programmy dlja JeVM [Certificate of state registration of computer programs] №2016661676. - Moscow: Rospatent, 2016.

20. Avtomatizirovannyj konverter modelej IDEF0 v diagrammy dejatel'nosti jazyka UML [Automated Converter of IDEF0 models to UML activity diagrams] «ToADConverter» («ToADConverter») / Authors-copyright holders: $\mathrm{Hu}-$ baev G.N., Shirobokova S.N., Tkachenko Ju. V., Titarenko E.V. // GeBIT 2015 (Gannover, 2015). Katalog razrabotok rossijskih kompanij [Catalog of developments of Russian companies]. - Ministry of Education and Science of the Russian Federation; MSP ITT. - 2015.

21. Hubaev G.N., Shherbakov S.M., Aruchidi N.A., Lubjanskij V.K. Sravnitel'naja ocenka sostava operacij delovyh processov (SOSOP) [Comparative evaluation of operation of business processes (SOAP)] // Svidetel'stvo o gosudarstvennoj registracii programmy dlja JeVM [Certificate of state registration of computer programs] №2017614714. - Moscow: Rospatent, 2017.

22. Khubaev G. N. Method of isolating a desired subset of objects from a set of greater power [Electronic resource] // Scientific research of the SCO countries: synergy and integration (Beijing, China 31 July 2019). - Beijing, 2019. P. 50-57. - URL: https://doi.org/10.34660/ INF.2019.13.33962.

23. Khubaev G.N., Scherbakov S.M., Shirobokova S. N. Conversion of IDEF3 models into UML-diagrams for the simulation in the SIM system-UML // European Science Review. 2015. — №12. — P. 20-25. 


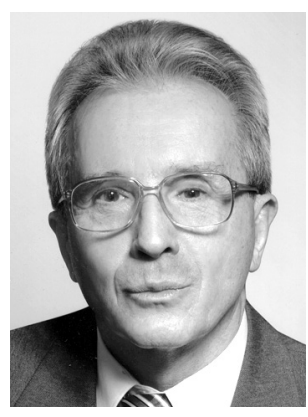

Хубаев Георгий Николаевич - доктор экономических наук, кандидат технических наук, профессор, профессор кафедры Информационных систем и прикладной информатики Ростовского государственного экономического университета (РИНХ).

Khubaev Georgy Nikolaevich - Doctor of Economic Sciences, Candidate of Engineering Sciences, Professor, Professor of the Department of Information Systems and Applied Informatics, Rostov State University of Economics (RSUE).

344002, г. Ростов-на-Дону, ул. Б. Садовая, 69

69 B. Sadovaya st., 344002, Rostov-on-Don, Russia

E-mail: gkhubaev@mail.ru 